\title{
Ifi204/p204, a new piece in the sepsis puzzle
}

\author{
Gayatri Subramanian $^{1}$, Ritu Chakravarti ${ }^{2}$, Saurabh Chattopadhyay ${ }^{1}$ \\ ${ }^{1}$ Department of Medical Microbiology and Immunology, ${ }^{2}$ Department of Physiology and Pharmacology, University of Toledo College of Medicine \\ and Life Sciences, Toledo, OH, USA \\ Correspondence to: Saurabh Chattopadhyay. Department of Medical Microbiology and Immunology, University of Toledo College of Medicine and \\ Life Sciences, Toledo, OH, USA. Email: Saurabh.Chattopadhyay@utoledo.edu. \\ Comment on: Yi YS, Jian J, Gonzalez-Gugel E, et al. p204 Is Required for Canonical Lipopolysaccharide-induced TLR4 Signaling in Mice. \\ EBioMedicine 2018;29:78-91.
}

Submitted Aug 28, 2018. Accepted for publication Sep 06, 2018.

doi: $10.21037 / \mathrm{atm} .2018 .09 .22$

View this article at: http://dx.doi.org/10.21037/atm.2018.09.22

Innate immunity is the first line of defense against microbial infection. The host cells are equipped with cellular sensors, which detect various components of microbes to mount rapid and early antimicrobial immune responses (1-3). The Toll-like receptors (TLRs) are transmembrane proteins, located on the cell surface or the endosome, to recognize the extracellular microbial components, and trigger proinflammatory and antimicrobial responses (1,3-5). Among the thirteen known TLRs, TLR4 is expressed both on the plasma, and the endosomal, membranes to detect the extracellular bacterial lipopolysaccharides (LPS) $(6,7)$. LPS recognition by the plasma membrane-bound TLR4 triggers intracellular signaling pathways by recruiting MyD88, a cytoplasmic adaptor protein. The TLR4-MyD88 complex activates, via a series of signaling proteins and kinases, the transcription factor NF- $\mathrm{KB}$ to induce the pro-inflammatory cytokines $(8,9)$. Extracellular LPS is also recognized by the endosomal TLR4, which recruits the cytoplasmic adaptor protein TRIF to activate NF- $\mathrm{kB}$ as well as IRF3, another transcription factor $(8,10)$. TLR4-TRIF signaling induces, in addition to the $\mathrm{NF}-\kappa \mathrm{B}-$ dependent proinflammatory cytokines, Type-I IFNs, e.g., IFN- $\beta$ by the coordinated action of both NF-KB and IRF3 (Figure 1). The uncontrolled expression of TLR4-induced genes leads to a pathological state known as sepsis $(6,11)$.

Bacterial sepsis is detrimental to the host and, therefore, the discovery of new proteins that regulate TLR4 signaling is highly significant. In a recent study, Liu and his colleagues described a new molecular mechanism by which Ifi204/ p204 contributes to TLR4 signaling and subsequently promotes sepsis in mice (12). Ifi204/p204 is a member of the p200 family of proteins, which are IFN-stimulated gene (ISG) products containing the Pyrin (PYD) and HIN-200 domains $(13,14)$. The p200 family of proteins are involved in a variety of functions including cellular differentiation, antiviral defense, and inflammasome activation (13-17). These proteins bind directly to the cytoplasmic nucleic acids to activate intracellular signaling pathways. Among the human p200 family of proteins, IFI16 recognizes cytoplasmic double-stranded (ds) DNA to induce Type-I IFN (18). AIM2, another human p200 protein, is involved in inflammasome activation in virus or bacteria-infected cells $(15,19)$. Ifi204/p204, the murine homolog of human IFI16, co-operates with cGAS, a cytoplasmic DNA sensor, to induce Type-I IFN in response to bacterial infection (20). However, the in vivo role of p204 remained unclear due to the absence of $p 204^{-/-}$animals. Yi et al. reported the first generation of $p 204^{-/-}$mice using the traditional geneediting technology (12). Using cells derived from the $p 204^{-1-}$ mice, the authors demonstrated that p204 is essential for LPS-activated TLR4 signaling and gene induction in the macrophages, which are critical cells that contribute to sepsis. The $p 204^{-1-}$ murine macrophages exhibit a strong reduction in LPS-induced pro-inflammatory cytokines, e.g., TNF- $\alpha$, IL- 6 , and IL- $1 \beta$ as well as Type-I IFNs, e.g., IFN- $\beta$. The $p 204^{-/-}$mice, when exposed to LPS, display a significant reduction in TLR4-induced genes. As a result of the reduced cytokine levels in the sera, the $p 204^{-/}$ mice are significantly resistant to LPS-induced sepsis. These results revealed a new function of p204 in LPSinduced sepsis both ex vivo and in vivo. LPS, in addition to stimulating TLR4, is also internalized by the macrophages 


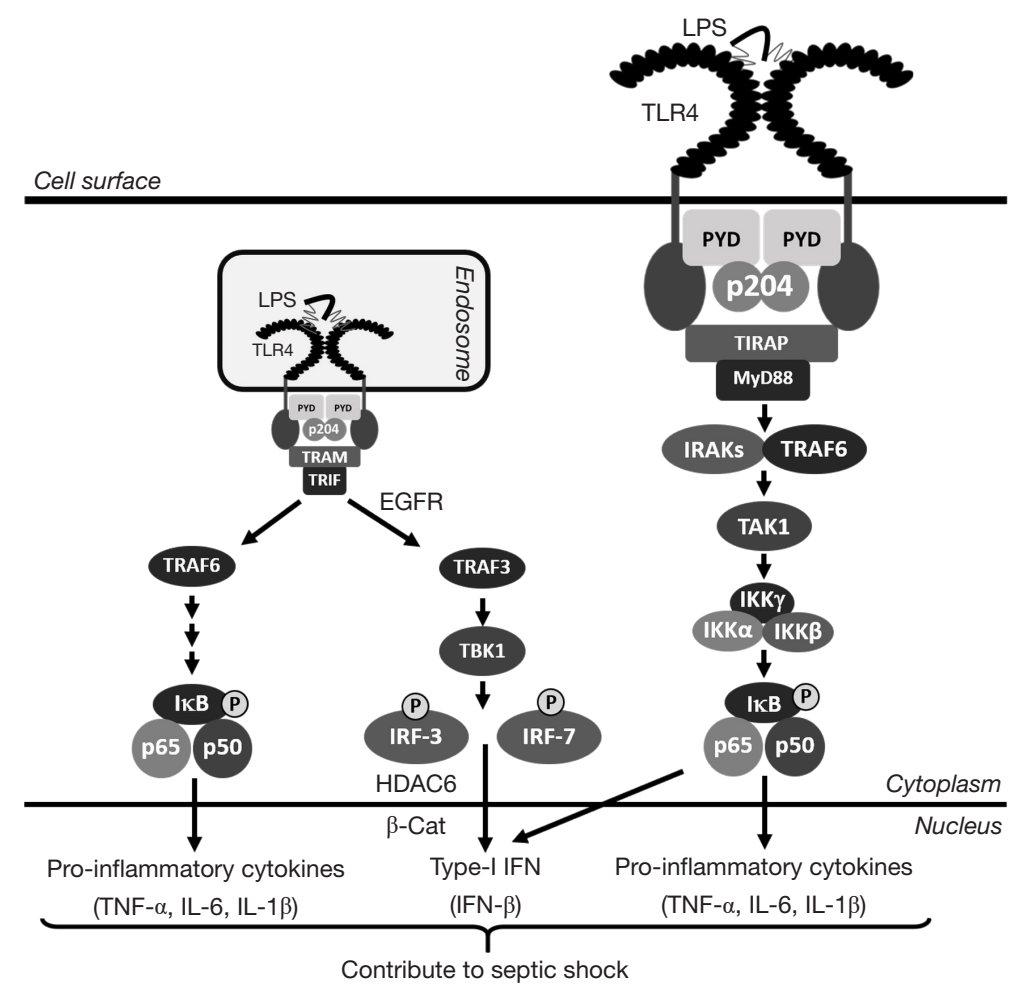

Figure 1 Ifi204/p204 forms TLR4 dimers to activate downstream signaling. LPS stimulates TLR4 on the cell surface and the endosome. TLR4-mediated downstream signaling is dependent on receptor dimerization, which is mediated by the pyrin domains (PYD) of Ifiz04/ p204. TLR4 signaling triggers the induction of pro-inflammatory cytokines and IFN- $\beta$. Unregulated expression of TLR4-induced genes leads to lethal septic shock.

to activate the intracellular LPS sensors. However, $p 204^{-1-}$ macrophages are not deficient in inflammasome activation in response to intracellular LPS. Therefore, p204 is neither a sensor for intracellular LPS nor a component of the intracellular LPS-activated inflammasome pathway. These results led to the identification of a direct role of p204 in TLR4 activation. TLR4, upon binding to LPS, undergoes dimerization, a step that is essential for the recruitment of MyD88 or TRIF. The $p 204^{-/-}$cells display a strong reduction in LPS-induced TLR4 dimerization. The PYD of p204 directly interacts with TLR4 to facilitate TLR4 dimerization. Furthermore, the PYD contains a binding motif (RKR), which is essential for p204-TLR4 interaction and LPS-induced TLR4 dimerization. As a result, the $p 204^{-1-}$ macrophages are defective in TLR4-mediated activation of $\mathrm{NF}-\kappa \mathrm{B}$ and IRF3, the transcription factors responsible for the induction TNF- $\alpha$, and IFN- $\beta$, respectively. The authors propose a model, in which p204 binds to TLR4 using its PYD to trigger LPS-mediated receptor dimerization and downstream signaling (Figure 1).
How the host mounts and regulates the innate immune responses is an area of intense investigation. The new mechanisms allow the successful design of therapeutics to target specific components of the innate signaling pathways (11). TLR4 signaling, on the one hand, contributes to the antimicrobial defense by triggering the synthesis of pro-inflammatory and antimicrobial genes. However, unregulated TLR4 activation leads to the pathological septic shock response. Moreover, TLR4 signaling in the Kupffer cells, activated by gut bacteriaderived LPS, contributes to the alcoholic liver diseases (21). Work through our research revealed that EGFR activity is required specifically for the endosomal TRIF-dependent, but not the cell surface MyD88-dependent, branch of TLR4 signaling (7). As a result, EGFR inhibitors protect against LPS-induced bacterial sepsis in mice $(7,22)$. EGFR activity is also required for the activation of TLR3, and TLR9, the endosomal TLRs $(23,24)$. In addition to EGFR, we have uncovered new components of TLR4 signaling; using knockout cells and mice, we showed that HDAC6 
and $\beta$-catenin are critical for LPS/TLR4 signaling (25). Ifi204/p204 is a new component of LPS/TLR4 signaling in macrophages (12). In the future, studies will be directed to understand the role of p204 in other TLRs, which activate similar downstream signaling pathways. Because dimerization is essential for all TLRs, it would be interesting to investigate the generality or the specificity of p204 involvement. The identification of a domain of TLR4 that interacts with p204 will help address these questions. It will be mechanistically significant to determine whether IFI16, the human homolog of murine p204, also similarly participates in TLR4 signaling. Future studies are required, using macrophage-specific $p 204^{-/-}$mice, to explore the cell type-specific role of p204 in bacterial sepsis. These studies will help develop therapeutics by targeting TLR4/p204 interaction for the treatment against sepsis.

\section{Acknowledgements}

Funding: Research in the authors' laboratories is supported by the National Institutes of Health grant AA026017 (SC), American Heart Association Grants 15SDG25090212 (SC), 15SDG2308025 (RC), and the University of Toledo College of Medicine and Life Sciences startup funds (SC).

\section{Footnote}

Conflicts of Interest: The authors have no conflicts of interest to declare.

\section{References}

1. Chow J, Franz KM, Kagan JC. PRRs are watching you: Localization of innate sensing and signaling regulators. Virology 2015;479-80:104-9.

2. Kumar S, Ingle H, Prasad DV, et al. Recognition of bacterial infection by innate immune sensors. Crit Rev Microbiol 2013;39:229-46.

3. Kawai T, Akira S. Toll-like receptors and their crosstalk with other innate receptors in infection and immunity. Immunity 2011;34:637-50.

4. Pasare C, Medzhitov R. Toll-like receptors: linking innate and adaptive immunity. Adv Exp Med Biol 2005;560:11-8.

5. Chattopadhyay S, Sen GC. Tyrosine phosphorylation in Toll-like receptor signaling. Cytokine Growth Factor Rev 2014;25:533-41.

6. Rosadini CV, Kagan JC. Early innate immune responses to bacterial LPS. Curr Opin Immunol 2017;44:14-9.
7. Chattopadhyay S, Veleeparambil M, Poddar D, et al. EGFR kinase activity is required for TLR4 signaling and the septic shock response. EMBO Rep 2015;16:1535-47.

8. Kenny EF, O'Neill LA. Signalling adaptors used by Tolllike receptors: an update. Cytokine 2008;43:342-9.

9. O'Neill LA, Fitzgerald KA, Bowie AG. The Toll-IL-1 receptor adaptor family grows to five members. Trends Immunol 2003;24:286-90.

10. Hoebe K, Beutler B. LPS, dsRNA and the interferon bridge to adaptive immune responses: Trif, Tram, and other TIR adaptor proteins. J Endotoxin Res 2004;10:130-6.

11. Hennessy EJ, Parker AE, O'Neill LA. Targeting Toll-like receptors: emerging therapeutics? Nat Rev Drug Discov 2010;9:293-307.

12. Yi YS, Jian J, Gonzalez-Gugel E, et al. p204 Is Required for Canonical Lipopolysaccharide-induced TLR4 Signaling in Mice. EBioMedicine 2018;29:78-91.

13. Lengyel P, Liu CJ. The p200 family protein p204 as a modulator of cell proliferation and differentiation: a brief survey. Cell Mol Life Sci 2010;67:335-40.

14. Luan Y, Lengyel P, Liu CJ. p204, a p200 family protein, as a multifunctional regulator of cell proliferation and differentiation. Cytokine Growth Factor Rev 2008;19:357-69.

15. Fernandes-Alnemri T, Yu JW, Juliana C, et al. The AIM2 inflammasome is critical for innate immunity to Francisella tularensis. Nat Immunol 2010;11:385-93.

16. Liu CJ, Wang H, Lengyel P. The interferon-inducible nucleolar p204 protein binds the ribosomal RNA-specific UBF1 transcription factor and inhibits ribosomal RNA transcription. EMBO J 1999;18:2845-54.

17. Stein SC, Falck-Pedersen E. Sensing adenovirus infection: activation of interferon regulatory factor 3 in RAW 264.7 cells. J Virol 2012;86:4527-37.

18. Almine JF, O'Hare CA, Dunphy G, et al. IFI16 and cGAS cooperate in the activation of STING during DNA sensing in human keratinocytes. Nat Commun 2017;8:14392.

19. Rathinam VA, Jiang Z, Waggoner SN, et al. The AIM2 inflammasome is essential for host defense against cytosolic bacteria and DNA viruses. Nat Immunol 2010;11:395-402.

20. Storek KM, Gertsvolf NA, Ohlson MB, et al. cGAS and Ifi204 cooperate to produce type I IFNs in response to Francisella infection. J Immunol 2015;194:3236-45.

21. Gao B, Seki E, Brenner DA, et al. Innate immunity in alcoholic liver disease. Am J Physiol Gastrointest Liver Physiol 2011;300:G516-25.

22. De S, Zhou H, DeSantis D, et al. Erlotinib protects 
against LPS-induced endotoxicity because TLR4 needs EGFR to signal. Proc Natl Acad Sci U S A 2015;112:9680-5.

23. Veleeparambil M, Poddar D, Abdulkhalek S, et al. Constitutively Bound EGFR-Mediated Tyrosine Phosphorylation of TLR9 Is Required for Its Ability To Signal. J Immunol 2018;200:2809-18.

24. Yamashita M, Chattopadhyay S, Fensterl V, et al. Epidermal growth factor receptor is essential for Toll-like receptor 3 signaling. Sci Signal 2012;5:ra50.

25. Chattopadhyay S, Fensterl V, Zhang Y, et al. Inhibition of viral pathogenesis and promotion of the septic shock response to bacterial infection by IRF-3 are regulated by the acetylation and phosphorylation of its coactivators. MBio 2013;4:e00636.
Cite this article as: Subramanian G, Chakravarti R, Chattopadhyay S. Ifi204/p204, a new piece in the sepsis puzzle. Ann Transl Med 2018;6(Suppl 1):S12. doi: 10.21037/ atm.2018.09.22 